\title{
Reverse remodeling in new onset heart failure and its affection on the right side parameters.
}

\author{
Manal Adel ${ }^{1}$, Yasser Sharaf ${ }^{1},{\text { Hesham } \text { Yehia }^{1} \text {, and Kareem Mahmoud }}^{1}$ \\ ${ }^{1}$ Cairo University Kasr Alainy Faculty of Medicine
}

June 3,2021

\begin{abstract}
Background Patient with new onset heart failure showed improvement in their EF and strain pattern along with improvement in cardiac biomarkers followed intervention procedures or device implantation and medical treatment. However right side changes are affected by these changes as well which needed further studies. Methods Hundred patients were included in a period starting from 2018 to 2020 and their follow up after 3-6 months :All patients underwent full assessment including Minnesota questionnaire of heart failure, examination and echocardiography with focusing on global longitudinal , circumferential strain ,right side areas and fractional area changes. Troponin I was evaluated at admission and follow up. Results There was significant change between patients at admission and follow up in Minnesta Q questionnaire from19.1 \pm 11.6 to $13.8 \pm 10.4$ with $\mathrm{P}$ value (0.001) , and change in troponin I 1.84 .9 to 0.10 .3 with P value $<0.001$. The Ejection fraction by 2D Simpson method showed statistically significant change from $30.6 \pm 8.5$ to $41.9 \pm 14.0$ with $\mathrm{P}$ value $<0.001$, Moreover ESV index showed a significant decrease from $50.5 \pm 21.1$ to $37.5 \pm 23.2$ with $\mathrm{P}$ value of 0.017 . Global longitudinal strain showed significant increase from $5.9 \pm 3.5$ to $7.5 \pm 3.8$ with $\mathrm{P}$ value 0.024 . The right side dimensions of end-systolic showed a significant increase from $9.8 \pm 4.5$ to 8.2 \pm 3.4 with $\mathrm{P}$ value 0.008 .FAC showed a significant increase from a mean of $41.7 \pm 10.7$ to $45.4 \pm 8.8$ with $\mathrm{P}$ value of 0.008 . Conclusion: Significant changes affected the cardiac volumes parameters in reverse remodeled followed medical treatment and/ or revascularization affecting both left and right side parameters..
\end{abstract}

\section{Introduction}

Remodeling is resulting in molecular, cellular and interstitial changes and manifested clinically as changes in size, shape and function of the heart resulting from cardiac load or injury. Cardiac remodeling is influenced by hemodynamic load, neurohormonal activation, and other factors still under investigation. Medical and device therapies that reduce heart failure morbidity and mortality also lead to decreased left ventricular volume and mass and a more normal elliptical shape of the ventricle. These are due to changes in myocyte size, structure, and organization that have been referred to collectively as reverse remodeling. Moreover, there are subsets of patients whose hearts have undergone reverse remodeling either spontaneously or after medical or device therapies and whose clinical course is associated with freedom from future heart failure events. However right side changes do occur along with reverse remodeling but still under investigation.

\section{Methods}

100 patients were included in a period starting from 2018 to 2020 and their follow up after 3-6 months :All patients underwent full study including Minnesota questionnaire of heart failure ,full study echocardiography including global longitudinal and circumferential strain ,right side volumes and fractional area change. Troponin I was withdrawn at admission and follow up.

46 were done follow up, 20 deaths and1 developed stroke 33 lost follow up.'13 patients were AF and the 
rest were sinus . 15 patients among the follow up underwent revascularization through PCI or CABG while others follow medical treatment and one patient had ICD :

\section{Study design}

The study protocol was approved by the ethics committee of the faculty of Medicine,Cairo University it was a prospective observational study on reverse remodeling in new onset heart failure patients with $\mathrm{EF}<40 \%$ . There is no universal imaging definition of reverse remodeling, but many studies have used statistically significant increases in LVEF or decreases in LV dimensions or volumes, while others have defined cut-offs for increases in LVEF (by 5-20 \%) and/or decreases in LV dimensions or volumes (by 10-20\%) with a change [?]15\% often being considered significant

\section{Inclusion and exclusion criteria}

Patients with recent onset (within three months) heart failure with reduced ejection fraction (less than 45\%).

Patients with peripartum heart failure, cardiogenic shock, chronic heart failure, Anthracyclin-induced heart failure, patient with Acute STEMI , patients who refuse to participate in this study, and Patients with poor acoustic window will be excluded from the study.

Full history and examination, Q minneosta questionnaire, troponinI was withdrawn and they all underwent echocardiography with speckle tracking on the left side, right side volumes and fractional area change and follow -up after 3-6 months., where all values with indexed to body surface

\section{Collection of data}

Kasralainy Cardiology department Cairo University 2018 to 2020 all patients underwent detailed full history, examination, cardiac troponin I along with routine labs and full study echocardiography including global longitudinal and circumferential strain and measurement of right side dimensions and FAC :All values were indexed to body surface area

\section{Study outcome}

Primary outcome between reverse remodeling and global longitudinal strain, LV diameters and volume index , and cardiac Troponin I.

\section{Secondary outcome}

Relation between reverse remodeling and right side dimensions and fractional area change.

\section{Statistical analysis}

Data were coded and entered using the statistical package for the Social Sciences (SPSS) version 26 (IBM Corp., Armonk, NY, USA). Data was summarized using mean, standard deviation, median, minimum and maximum in quantitative data and using frequency (count) and relative frequency (percentage) for categorical data. Comparisons between quantitative variables were done using the non-parametric Mann-Whitney test. For comparison of serial measurements within each patient the non-parametric Wilcoxon signed rank test was used (Chan, 2003a) ${ }^{(1)}$. For comparing categorical data, Chi square $(\chi 2)$ test was performed. Exact test was used instead when the expected frequency is less than 5 (Chan, 2003b $)^{(2)}$. Correlations between quantitative variables were done using Spearman correlation coefficient (Chan, 2003c $)^{(3)}$. Logistic regression was done to detect independent predictors of mortality $\left(\right.$ Chan, 2004) ${ }^{(4)}$. P-values less than 0.05 were considered as statistically significant.

\section{Results}

\section{Patient related variables}

Mean age, weight, height of our population study were $50.8 \pm 15.3,81.4 \pm 19.7$, and $1.6 \pm 0.1$ respectively.Male patients were $71 \%$, diabetes were $39 \%$ and hypertensive were $35 \%$ respectively, $70 \%$ were ischemia and $19 \%$ were DCM.. 


\section{Discussion:}

Our study included 100 patients 45 patients completed follow up 3-6 months (90-180 Days) and 20 died .70\% of the patients were of ischemic etiology $15 \%$ underwent complete revascularization while the rest continue follow up. 12(26\%) patients showed RR after revascularization and 21(46\%) patients showed RR followed medical treatment with Q Minnesota questionnaire of heart value of $\mathrm{P}$ value significance $<0.001$. and Heart rate changed from mean $93 \pm 19.4$ to $82.8 \pm 30$.1 beats per minute with significant $\mathrm{p}$ value $<0.007$. Change of MR $1.4 \pm 2.8$ to $1.2 \pm 2.7$ with $\mathrm{P}$ value 0.127 (it was not statistically significant), ejection fraction $30.6 \pm 8.5$ to mean $41.9 \pm 14.0$ with $\mathrm{P}$ value $<0.001$ and $\mathrm{ESV}$ index from $50.5 \pm 21.1$ to $37.5 \pm 23.2$ with $\mathrm{p}$ value 0.017 and Global longitudinal strain mean value $5.9 \pm 3.5$ to $7.5 \pm 3.8$ with $\mathrm{P}$ value 0.024 ,. The right side dimensions of end-systolic showed a significant increase from $9.8 \pm 4.5$ to $8.2 \pm 3.4$ with $\mathrm{P}$ value 0.008 .FAC showed a significant increase from a mean of $41.7 \pm 10.7$ to $45.4 \pm 8.8$ with $\mathrm{P}$ value of 0.008 .

100 new onset heart failure patients were included with 45 underwent follow up ,33 showed reverse remodeling and 20patients died, Troponin I was statistically significant with mean $1.8 \pm 4.9$ to $0.1 \pm 0.3$ with $\mathrm{P}$ value $<0.001$ for patients with ejection fraction $30.6 \pm 8.5$ to mean41.9 \pm 14.0 with $\mathrm{P}$ value $<0.001$, Troponin I change mean $0.15 \pm 0.2$ to $0.13 \pm 0.2$ among group 1 and 3 respectively with $\mathrm{P}$ value 0.710 . These values showed the significant in cardiac troponin and its affection by reverse remodeling of the heart failure patients with improvement in ejection fraction and reduction of volume of LV cavity .

Right ventricular function has a dichotomous role. At diagnosis it is an important prognostic marker in $\mathrm{DCM}^{(5,7)}$. Interestingly, it frequently shows a rapid recovery under therapy (up to 6 months). Right ventricular function normalization is part of a global hemodynamic improvement induced by therapy and precedes LVRR. It is emerging as an early therapeutic target and an independent prognostic predictor ${ }^{(5)}$. Improvement in right ventricular function is also described in CRT implanted patients, probably due to a hemodynamic improvement very early after resynchronization, and it is associated with an improvement in survival rates ${ }^{(6)}$. Conversely, the development of right ventricular dysfunction during follow-up is an expression of structural progression of the disease and portends a negative outcome ${ }^{(5)}$.

From 1993 to 2008, we analyzed 512 patients with DCM (46 years of age [36 to 55 years of age], left ventricular ejection fraction $32 \%$ [25\% to 41\%]) with a potential follow-up of [?]72 months and available data at baseline and at least 1 pre-specified follow-up evaluation (i.e., 6, 24, 48, or 72 months). RV dysfunction was defined as RV fractional area change $<35 \%$ at 2 -dimensional echocardiography. The primary outcome measure was a composite of death or heart transplantation.

At enrollment, 103 (20\%) patients had RV dysfunction. During follow-up, 89 of them $(86 \%, 17 \%$ of the overall cohort) normalized RVF at a median time of 6 months, whereas 38 of the remaining 409 patients with normal baseline RVF (9\%; 7\% of the overall population) exhibited a new-onset RV dysfunction (median time: 36 months). RVF normalization was significantly associated with subsequent left ventricular reverse remodeling that was observed at a median time of 24 months (odds ratio: 2.49; $95 \%$ confidence interval [CI]: 1.17 to $5.3 ; \mathrm{p}=0.018$ ). At baseline multivariate analysis, RV dysfunction was independently associated with the primary outcome measure (hazard ratio: $1.71 ; 95 \% \mathrm{CI}: 1.02$ to $2.85 ; \mathrm{p}=0.0413$ ). At time-dependent model, RVF revaluation over time maintained an independent predictive value (hazard ratio: 2.83; 95\% CI: 1.57 to $5.11 ; \mathrm{p}=0.0006)$.

\section{Study limitations:}

1.Sample size relatively small

2.Patients who lost follow up affected the study results.

3. Patient who died early after 3-6 months.

\section{Conclusion:}

Reverse remodeling is affected by cardiac biomarkers Troponin I. . 
Significant changes affected the cardiac volumes parameters in remodeled heart compared to non remodeled followed medical treatment and or revascularization affecting both left and right side of heart.

Speckle tracking global longitudinal strain is a prognostic marker in reverse remodeling heart failure with reduced ejection fraction.

Recommendation:

Reveres remodeling is needed to be evaluated in different etiology of heart failure and in those who continue medical treatment without devices or intervention in future studies.

Abbreviations

GLS global longitudinal strain, EF ejection fraction, ESVi end-systolic volume index, FAC fractional area change, DCM dilated cardiomyopathy, RVF right ventricle function, RV right ventricle.

\section{Acknowledgement}

I would like to thank all one for participating in this paper research and work as one team to present this research thoroughly.

Author Contributions

Dr Hesham Yehia and Dr Yasser Sharaf revised the research

Consent for publication

All authors gave their consent for publication

Funding

Self funded

Conflict of interest

None

References:

1. Chan, Y. H. (2003). Biostatistics 102: quantitative data-parametric \& non-parametric tests. blood Press , $140(24.08), 79$.

2 Chan, Y. H. (2003). Biostatistics 103: qualitative data-tests of independence. Singapore Med J , 44 (10), 498-503..

3. Chan, Y. H. (2003). Biostatistics 104: correlational analysis. Singapore Med J , 44 (12), 614-9..

4. Chan, Y. H. (2004). Biostatistics 202: logistic regression analysis. Singapore medical journal , 45 (4), 149-153.

5. Merlo, M., Gobbo, M., Stolfo, D., Losurdo, P., Ramani, F., Barbati, G., .. \& Sinagra, G. (2016). The prognostic impact of the evolution of RV function in idiopathic DCM. JACC: Cardiovascular Imaging , 9 (9), 1034-1042.

6. Stolfo, D., Merlo, M., Pinamonti, B., Poli, S., Gigli, M., Barbati, G., .. \& Sinagra, G. (2015). Early improvement of functional mitral regurgitation in patients with idiopathic dilated cardiomyopathy. The American Journal of Cardiology , 115 (8), 1137-1143.

7. Merlo, M., Caiffa, T., Gobbo, M., Adamo, L., \& Sinagra, G. (2018). Reverse remodeling in dilated cardiomyopathy: insights and future perspectives. IJC heart $\mathscr{E}$ vasculature , 18 , 52-57. 